\title{
miRNA-1284 inhibits cell growth and induces apoptosis of lung cancer cells
}

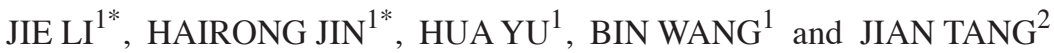 \\ ${ }^{1}$ Department of Thoracic Surgery, Chinese PLA General Hospital, Beijing 100853; ${ }^{2}$ Department of Cardio-Thoracic Surgery, \\ First Affiliated Hospital of Chinese PLA General Hospital, Beijing 100048, P.R. China
}

Received April 6, 2016; Accepted March 7, 2017

DOI: $10.3892 / \mathrm{mmr} .2017 .6949$

\begin{abstract}
Lung cancer is the most common cancer worldwide, and morbidity and mortality associated with lung cancer has been increasing annually in recent decades. MicroRNAs (miRNAs), which are short non-coding RNA sequences that are involved in the regulation of gene expression, have been previously demonstrated to be key regulators in cancer. The present study aimed to clarify the role of miRNA (miR)-1284 in lung cancer. A549 lung carcinoma cells were transfected with miR-1284 mimic or miR-1284 inhibitor using Lipofectamine 2000. Subsequently, cell viability, growth and apoptosis of A459 cells in the miR-1284 mimic, miR-1284 inhibitor and control groups were assayed by MTT assay, bromodeoxyuridine assay and flow cytometry, respectively. Furthermore, the protein expression levels of p27, p21, Bax, pro-caspase-3, activated caspase-3 and Myc were detected by western blot analysis to investigate the molecular mechanisms underlying the effect of miR-1284 on A549 cells. The cell viability and growth of A549 cells were significantly decreased in the miR-1284 mimic group compared with the control group, whereas the percentage of apoptotic cells was significantly increased. By contrast, miR-1284 inhibitor transfection significantly increased the cell viability and growth compared with control, and decreased apoptosis. Furthermore, expression of p27 was increased in miR-1284 mimic-transfected A549 cells compared with the control group, whereas p21 was unaffected by miR-1284 overexpression or inhibition. The expression of Myc was decreased by miR-1284 mimic transfection compared with the control group. For the other apoptosis-associated proteins that were investigated (Bax, pro-caspase-3 and active caspase-3), the expression levels in
\end{abstract}

Correspondence to: Dr Jian Tang, Department of Cardio-Thoracic Surgery, First Affiliated Hospital of Chinese PLA General Hospital, 51 Fucheng Road, Haidian, Beijing 100048, P.R. China

E-mail: tangjian19684@126.com

*Contributed equally

Key words: lung cancer, microRNA-1284, cell viability, apoptosis, $\mathrm{Myc}$ the miR-1284 mimic transfected cells were higher than in the other two groups (control and miR-1284 inhibitor). In conclusion, the results suggest that miR-1284 affects cell proliferation and apoptosis of lung cancer cells, indicating that miR-1284 may have a key role in lung tumorigenesis.

\section{Introduction}

Lung cancer is the leading cause of cancer-associated mortality with a consistently poor 5-year survival rate (1). Despite improvements in monitoring and clinical treatment strategies, the 5-year overall survival rate in the United States remains $\sim 16 \%$ (2). In 2012, lung cancer occurred in 1.8 million people and resulted in 1.6 million mortalities worldwide (3). Unfortunately, its total incidence is increasing dramatically worldwide (4,5). Most clinical cases of lung cancer are categorized into two subtypes: Small-cell lung carcinoma (SCLC) and non-small-cell lung carcinoma (NSCLC). Approximately $70 \%$ of patients newly diagnosed with either subtype of lung cancer present with locally recurrent or metastatic lesions following resection (6). Undoubtedly, elucidating the pathogenesis of lung cancer is important for the effective treatment of this disease.

MicroRNAs (miRNAs) are a class of short, endogenous, noncoding RNAs that regulate gene expression. Although miRNAs account for only a minor fraction of the expressed genome, they have been demonstrated to have important roles in various cellular processes, including proliferation, apoptosis, differentiation and malignant transformation (7-9). It is estimated that $60 \%$ genes are regulated by miRNAs, and changes in miRNA expression affect physiological and pathological processes (10). Increasing evidence indicates that miRNAs have critical functions in the initiation and progression of various malignancies, including lung cancer $(11,12)$. For instance, miRNA-145 (miR-145), miR-328, miR-378 and miR-21 have been reported to have important roles in lung cancer (13-15). Recently, miR-1284 was reported to be downregulated in gastric cancer, and miR-1284 overexpression promoted cell cycle arrested at the G0/G1 phase, accelerated drug-induced apoptosis, and decreased migration and invasiveness (16). To the best of our knowledge, the role of miR-1284 in lung cancer has not been previously investigated.

The present study aimed to investigate the role of miR-1284 in lung cancer. Therefore, A549 lung carcinoma cells were 
transfected with miR-1284 mimic or miR-1284 inhibitor. Cell viability, growth and apoptosis were then investigated under the two conditions. In order to further investigate the underlying molecular mechanisms, the protein expressions of cell cycle regulators (p27 and p21), apoptosis-associated proteins, including $\mathrm{Bcl}-2$ associated $\mathrm{X}$, apoptosis regulator (Bax), pro-caspase-3 and activated caspase-3, and Myc, were determined by western blot analysis. Results of the present study may lead to new treatment options for lung cancer.

\section{Materials and methods}

Cell culture. A549 human lung cancer cells were obtained from the American Type Culture Collection (Manassas, VA, USA) and cultured in Dulbecco's modified Eagle's medium (Invitrogen; Thermo Fisher Scientific, Inc., Waltham, MA, USA) supplemented with $10 \%$ fetal bovine serum (Invitrogen; Thermo Fisher Scientific, Inc.) at $37^{\circ} \mathrm{C}$ and $5 \% \mathrm{CO}_{2}$. The miR-1284 mimic (sense, UCUAUACAGACCCUGGCU UUUC; antisense, AAAGCCAGGGUCUGUAUAGAUU), miR-1284 inhibitor (GAAAAGCCAGGGUCUGUAUAGA) were synthesized by Shanghai GenePharma Co., Ltd. (Shanghai, China). The scramble miRNA control used was a 22 nucleotide random sequence with a non-targeting RNA duplex (GE Dharmacon; GE Healthcare Life Sciences, Little Chalfont, UK). The cells were transfected with $50 \mathrm{nM}$ miR-1284 mimic, miR-1284 inhibitor or scramble control by using Lipofectamine 2000 (Invitrogen; Thermo Fisher Scientific, Inc.) according to the manufacturer's manual. Cells were incubated at $37^{\circ} \mathrm{C}$ in a $\mathrm{CO}_{2}$ incubator for $24-48 \mathrm{~h}$.

RNA extraction and reverse transcription-quantitative polymerase chain reaction ( $R T-q P C R)$. At $48 \mathrm{~h}$ after transfection was complete, total RNA $\left(5 \times 10^{6}\right.$ cells) was extracted by TRIzol reagent (Invitrogen; Thermo Fisher Scientific, Inc.) and quantified by using agarose gel (1\%) electrophoresis and spectrophotometric analysis $\left(\mathrm{A}_{260} / \mathrm{A}_{280}\right.$ ratio). For cDNA synthesis, $2 \mu \mathrm{g}$ total RNA was reverse-transcribed by using a Transcriptor First-Strand cDNA Synthesis kit (Roche Molecular Systems, Inc., Pleasanton, CA, USA), according to the manufacturers protocol. The qPCR was performed by using FastStart Universal SYBR-Green Master (ROX; Roche Molecular Systems, Inc.) on the Applied Biosystems 7500 Fast Real-Time PCR system (Applied Biosystems, Thermo Fisher Scientific, Inc.). The thermocycling parameters were $95^{\circ} \mathrm{C}$ for $3 \mathrm{~min}$, followed by 40 cycles of $95^{\circ} \mathrm{C}$ for $15 \mathrm{sec}$ and by $60^{\circ} \mathrm{C}$ for $30 \mathrm{sec}$. Each sample was run in triplicate and was normalized to U6 small nuclear RNA levels. The following primers were used for qPCR: miR-1284, 5'-GGTCTATACAGACCCTGG CTTTTC-3' (forward) and 5'-CTCAACTGGTGTCGTGGA-3' (reverse); and U6, 5'-TTATGGGTCCTAGCCTGAC-3' (forward) and 5'-CACTATTGAGGGTATGC-3' (reverse). All primers were synthesized by Shanghai GenePharma Co., Ltd. Melting curve analysis was performed to confirm the specificity of the PCR products. The replicates were subsequently averaged, and fold induction was determined by a $\Delta \Delta \mathrm{Cq}$-based fold change calculation (17).

Cell viability assay. Cells in three groups (transfected with miR-1284, miR-1284 inhibitor and scramble control) were seeded into 96 -well culture plates at $5 \times 10^{3}$ cells/well and cultured for 1-5 days. Each group had three replicates. Cell viability was then assayed by adding $20 \mu 110 \mathrm{mg} / \mathrm{ml}$ 3-[4,5-dimethylthiazol-yl]-2,5-diphenyltetrazolium bromide (MTT; Sigma-Aldrich; Merck KGaA, Darmstadt, Germany), followed by incubation for $3 \mathrm{~h}$ at $37^{\circ} \mathrm{C}$. Then $150 \mu \mathrm{l}$ dimethyl sulfoxide was added to each well, and the optical density at $590 \mathrm{~nm}$ was recorded with a Multiskan EX (Thermo Fisher Scientific, Inc.). The results of three independent experiments are presented as the mean \pm standard deviation.

Bromodeoxyuridine (BrdU) assay. Cells were seeded in 6-well plates at a density of $2 \times 10^{4}$ cells/well on sterilized coverslips. At $72 \mathrm{~h}$ after transfection, BrdU (Sigma-Aldrich; Merck $\mathrm{KGaA}$ ) was added to the medium at a final concentration of $10 \mu \mathrm{M}$. After incubation at $37^{\circ} \mathrm{C}$ for $5 \mathrm{~h}$ in the dark, cells were fixed in cold $70 \%$ ethanol for $5 \mathrm{~min}$. DNA was denaturized by adding $1.5 \mathrm{M} \mathrm{HCl}$ to the cells for $30 \mathrm{~min}$ at room temperature. Cells were washed with $150 \mu \mathrm{l}$ PBS and $1 \%(\mathrm{v} / \mathrm{v})$ bovine serum albumin (Sigma-Aldrich; Merck KGaA) at room temperature for $20 \mathrm{~min}$ for blocking. Immunofluorescence to visualize incorporated $\mathrm{BrdU}$ was performed using a mouse anti-BrdU antibody (cat no. 560808; 1:2,000; BD Biosciences, San Jose, CA, USA) according to the manufacturer's instructions, and incubated for $1 \mathrm{~h}$ at room temperature. Then, secondary red-fluorescence dye-conjugated antibody Alexa Fluor 594 (ab150116; 1:500; Abcam, Cambridge, UK) was added and incubated for $45 \mathrm{~min}$ at room temperature. VECTASHIELD mounting medium with DAPI (Vector Laboratories, Burlingame, CA, USA) was used to stain the nuclei. Images were visualized using a Leica inverted fully automated microscope (DMI6000B) with digital camera DFC 420 RGB (Leica Microsystems GmbH, Wetzlar, Germany).

Apoptosis assay. The cells ( $24 \mathrm{~h}$ after transfection) were seeded into culture dishes at a density of $2 \times 10^{5}$ cells per culture dish. Then cells were incubated with Annexin V-fluorescein isothiocyanate (BD Biosciences) and propidium iodide (PI; BD Biosciences) for $30 \mathrm{~min}$ in the dark at $25^{\circ} \mathrm{C}$ to analyze the apoptotic percentage of cells. Cells were analyzed with a FACSCalibur flow cytometer (BD Biosciences) and the results were analyzed by using FlowJo 10 software (Tree Star, Inc., Ashland, OR, USA).

Western blot analysis. Cells (48 h after transfection) were washed once with PBS and lysed in RIPA buffer (Pierce; Thermo Fisher Scientific, Inc.). Protein samples were quantified with the Pierce BCA Protein assay kit (Pierce; Thermo Fisher Scientific, Inc.) and were then boiled for $10 \mathrm{~min}$ in sodium dodecyl sulfate (SDS) sample buffer. Equal amounts of protein (20 $\mu \mathrm{g} /$ well) were separated by $10 \%$ SDS-polyacrylamide gel electrophoresis and transferred to nitrocellulose membrane. After blocking with 5\% skim milk in $0.05 \%$ TBS-Tween-20 $(\mathrm{v} / \mathrm{v})$ for $1 \mathrm{~h}$ at room temperature, the membranes were incubated with the appropriate primary antibodies (at a dilution of $1: 1,000)$ overnight at $4^{\circ} \mathrm{C}$. Antibody specific to actin (C-2; cat no. 4967) was purchased from Cell Signaling Technology, Inc. (Danvers, MA, USA); c-Myc (cat no. ab32072), active caspase-3 (cat no. ab2302), p27 (cat no. ab54563), p21 (cat no. ab7960) and pro-caspase-3 (cat no. ab32150) were purchased from Abcam. Bax antibody (cat no. 2772) was purchased from Cell 


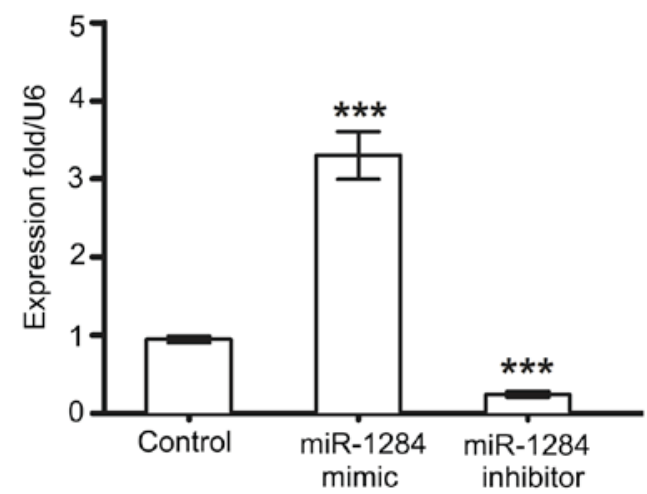

Figure 1. A549 cells were transfected with miR-1284 mimic, miR-1284 inhibitor, or scramble control. Expression of miR-1284 in A549 cells was measured by quantitative polymerase chain reaction. ${ }^{* * *} \mathrm{P}<0.001$ vs. control group. miR, microRNA.

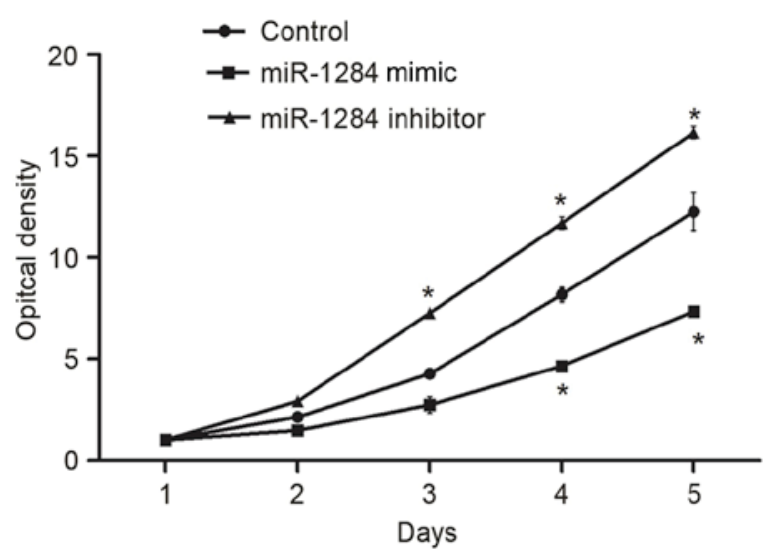

Figure 2. Cell viability assayed by MTT assay. Cell viability of A549 cells was decreased significantly upon miR-1284 overexpression while increased significantly upon miR-1284 inhibition. Values are presented as the mean \pm standard deviation. ${ }^{*} \mathrm{P}<0.05$ vs. control group at the same time point. miR, microRNA.

Signaling Technology, Inc. Then membranes were washed once with TBS-Tween-20 and incubated with anti-mouse and anti-rabbit horseradish peroxidase-conjugated secondary antibodies (cat nos. ab131368 and ab191866, respectively; 1:5,000) for $2 \mathrm{~h}$ at room temperature. Protein bands were visualized by using WEST-ZOL (plus) Western Blot Detection system (Intron Biotechnology, Inc., Seongnam, Korea).

Statistical analysis. Statistical analyses were performed using SPSS 19.0 statistical software (IBM SPSS, Armonk, NY, USA). All experiments were repeated three times. The results of multiple experiments were presented as the mean \pm standard deviation. The data were initially tested for normal distribution using one-sample Kolmogorov-Smirnov test, and then analyzed by one-way analysis of variance followed by Dunnett's test for multiple comparisons. $\mathrm{P}<0.05$ was considered to indicate a statistically significant difference.

\section{Results}

Role of miR-1284 in cell proliferation. Preliminary experiments to determine whether transfection with miR-1284 mimic and miR-1284 inhibitor successfully increased or decreased miR-1284 expression were performed, and the results indicated that transfection with miR-1284 mimic successfully increased the expression of miR-1284 and miR-1284 inhibitor transfection reduced levels, compared with control cells (Fig. 1). To determine the effect of miR-1284 on cell viability, A549 cells were transfected with miR-1284 or miR-1284 inhibitor and an MTT assay was performed. As demonstrated in Fig. 2, after transfection with miR-1284 mimic, the cell viability of A549 cells was decreased significantly compared with control $(\mathrm{P}<0.05)$. When cells were transfected with miR-1284 inhibitor, the cell viability was significantly increased compared with control cells $(\mathrm{P}<0.05)$.

Role of miR-1284 in cell growth. BrdU staining was used to determine the role of miR-1284 in cell growth. Compared with the control group, a 20.7\% decrease of BrdU-positive cells was detected in response to miR-1284 overexpression $(\mathrm{P}<0.05)$, whereas the miR-1284 inhibitor caused a $7.3 \%$ increase compared with the control group, however, this was not statistically significant (Fig. 3A and B).

To further understand the mechanisms of miR-1284 induced cell growth inhibition, we analyzed the effect of miR-1284 on cell cycle regulators in A549 cells. A strong increase in p27 was detected in response to the miR-1284 expression compared with the control. The other major cyclin-dependent kinase inhibitor 1 family member, p21, was unaffected by miR-1284 overexpression or inhibition (Fig. 3C).

Role of miR-1284 in apoptosis. To analyze the effect of miR-1284 on cell apoptosis, A549 cells were transfected with miR-1284 mimic or inhibitor and an apoptosis assay was performed. As presented in Fig. 4A and B, following transfection with miR-1284 mimic in A549 cells, the percentage of apoptotic cells was significantly increased from $8.0 \pm 1.0 \%$ (control group) to $27.6 \pm 3.2 \%(\mathrm{P}<0.05)$. However, when cells were transfected with miR-1284 inhibitor, the percentage $(3.09 \pm 0.6 \%)$ was decreased significantly compared with the control $(\mathrm{P}<0.05)$.

miR-1284 may induce apoptosis by targeting Myc. To further understand the mechanisms of miR-1284 on apoptosis, the protein expressions of Myc, Bax, pro-caspase- 3 and activated caspase- 3 in the three groups were detected by western blot. The results in Fig. 4C demonstrated that, compared with control, the expression of Myc decreased markedly in the miR-1284 group, and increased markedly in the miR-1284 inhibitor group. For the other three proteins, their expressions in the miR-1284 group were higher than in the other two groups.

\section{Discussion}

The results of the present study demonstrated that miR-1284 has an important role in cell viability, growth and apoptosis of A549 cells. Following transfection with miR-1284, cell viability and growth decreased significantly, while apoptosis was significantly increased. By contrast, miR-1284 inhibition significantly increased the cell viability and growth, and decreased apoptosis in A549 cells. Furthermore, the expression 
A

DAPI
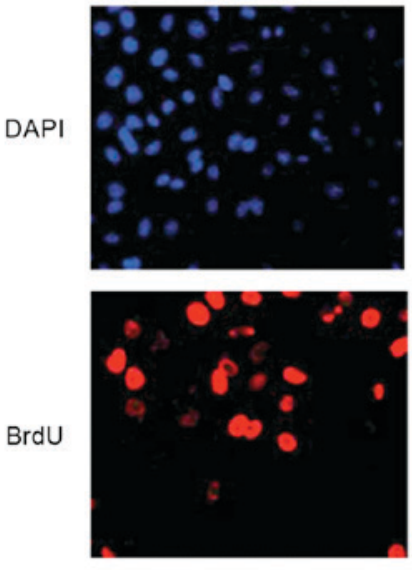

Control

B

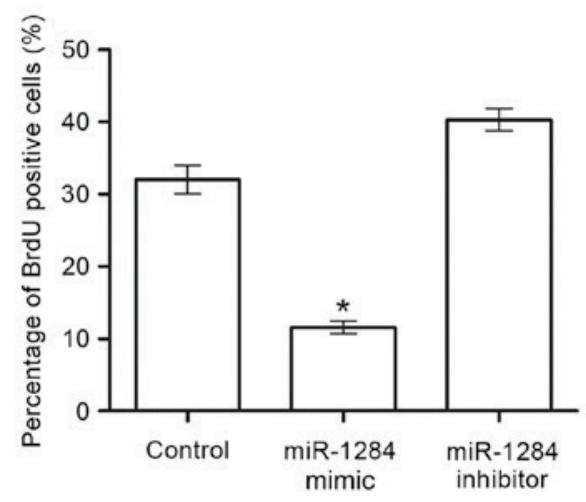

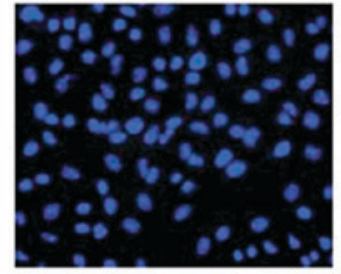
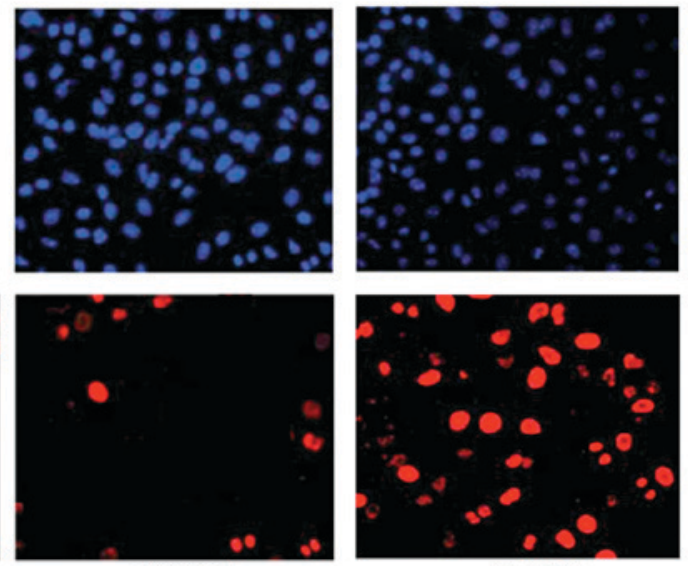

$\operatorname{miR}-1284$ mimic

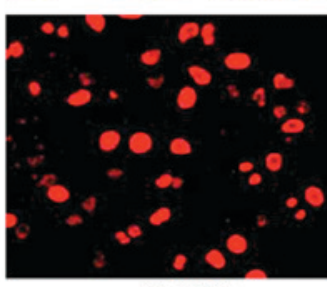

miR-1284 inhibitor

C

p27

p21

Actin
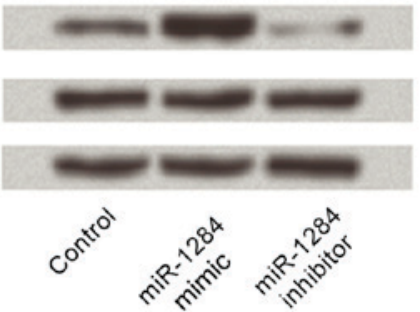

Figure 3. (A) Immunofluorescence analysis of BrdU incorporation in A549 cells transfected with miR-1284 mimic or miR-1284 inhibitor. BrdU was stained with AlexaFluor594-conjugated antibodies (red). Cell nuclei were visualized by DAPI staining. (B) Quantitation of BrdU staining results ( $\mathrm{n}=3$ ). (C) Protein expression of p27 and p21 detected by western blot analysis. Values are presented as the mean \pm standard deviation. " $\mathrm{P}<0.05$ vs. control group. BrdU, bromodeoxyuridine; miR, microRNA.

of a cell cycle regulator, p27, was markedly increased and the expression of Myc was markedly decreased when miR-1284 was overexpressed in A549 cells. For the other three apoptosis-associated proteins, their expressions in miR-1284 mimic group were higher than in the control and miR-1284 inhibitor groups.

A previous study has reported that altered expression of miRNAs targeting cyclins, cyclin-dependent kinases (CDKs) or CDK inhibitors, such as p21 and p27, is involved in the deregulation of cell cycle progression during tumorigenesis (18). The results of the present study indicated that the expression of p27 was increased when miR-1284 was overexpressed, whereas p21 was unaffected by miR-1284 overexpression or inhibition. p27 belongs to the Cip/Kip family of CDK inhibitor proteins, which bind to cyclin D alone or its catalytic subunit CDK4 to control the cell cycle progression at G1 (19). In accordance with this, the present study demonstrated that cell growth was decreased significantly by miR-1284 overexpression, indicating that miR-1284 may induce cell growth arrest by upregulating p27 expression in A549 cells.

Apoptosis is a complex biological process that enables organisms to kill and remove unwanted cells during their development, normal homeostasis and disease $(20,21)$. Evasion of apoptosis is a crucial step in the initiation and progression of cancer (22). The caspase family of cysteine proteases have key roles in the initiation and execution of apoptosis $(23,24)$. Caspase- 3 is a member of the caspase family that exists in the cell as a low-activity zymogen, pro-caspase-3. Pro-caspase-3 can be activated by proteolysis, and active caspase- 3 is necessary for initiation of apoptosis (25). In the current study, miR-1284 overexpression significantly increased the apoptosis rate of A549 cells. Furthermore, the expression of pro-caspase- 3 and active caspase- 3 was also increased by miR-1284, which indicated that miR-1284 may be involved in accelerating the apoptosis of A549 cells.

In addition to pro-caspase- 3 and active caspase- 3 , Bax expression was also increased by miR-1284 overexpression. Bax is a member of the Bcl-2 apoptosis regulator protein family, which has been studied intensively owing to their importance in the regulation of apoptosis and tumorigenesis (26). Bax is a pro-apoptotic protein and inactive in the cytosol of normal cells but becomes active in apoptotic cells by changing conformation in a multistep process (27). In accordance with this, the results of the present study also demonstrated that Bax was upregulated in apoptotic cells, further indicating that miR-1284 accelerates apoptosis.

Unlike the aforementioned proteins, the expression of Myc was decreased in the miR-1284 overexpression group and increased in the miR-1284 inhibitor group compared with control. Myc protein is a multifunctional, nuclear 

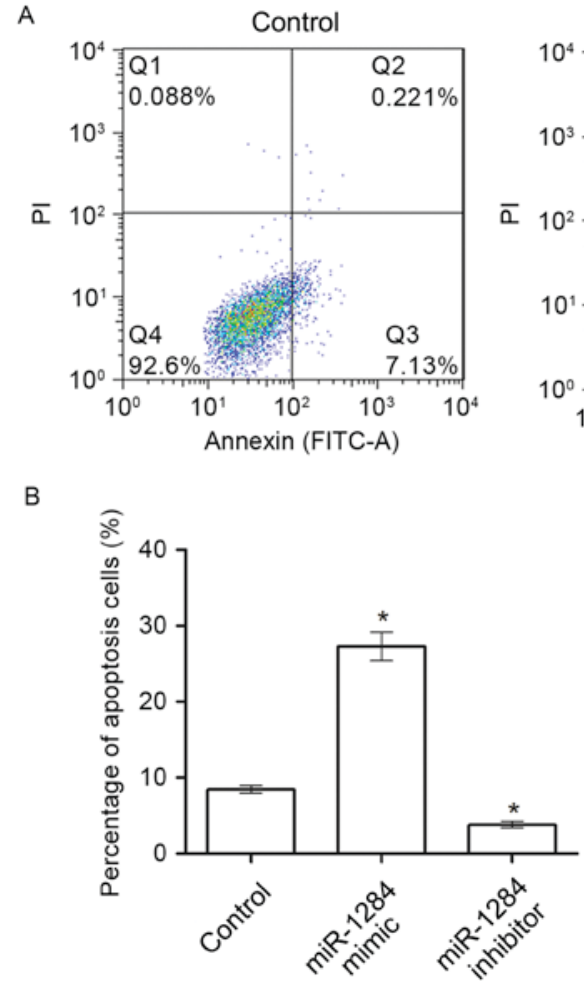
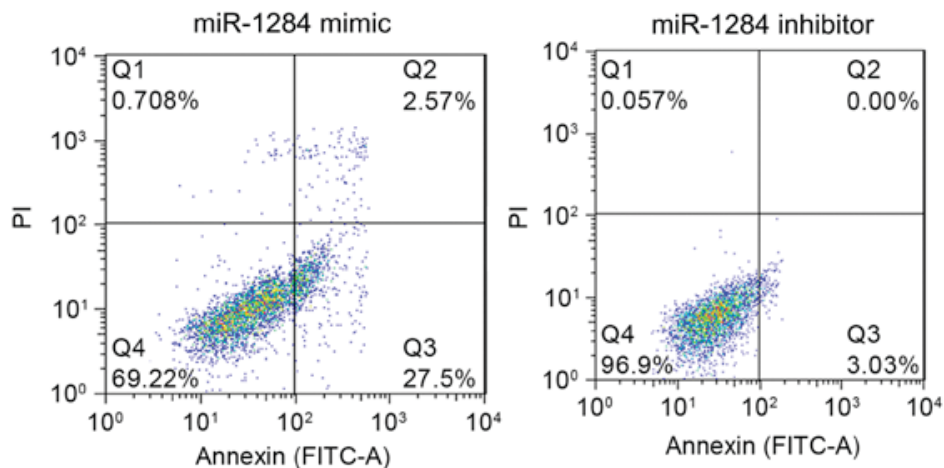

C

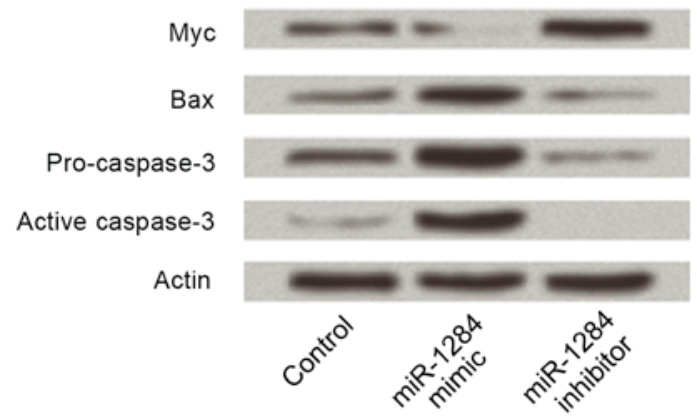

Figure 4. (A) Apoptotic cells were analyzed by flow cytometry to differentiate between apoptotic cells (annexin-V positive and PI-negative) and necrotic cells (annexin-V and PI-positive). Cells in Q3 were considered to be apoptotic cells and were compared between the groups. (B) Quantitation of flow cytometry apoptosis assay results. (C) Protein expression of Myc, Bax, pro-caspase-3 and active caspase-3 was determined by western blot analysis. Values are presented as the mean \pm standard deviation. ${ }^{*} \mathrm{P}<0.05$ vs. control group. miR, microRNA; PI, propidium iodide; FITC, fluorescein isothiocyanate; Bax, Bcl-2 associated X, apoptosis regulator; Q, quadrant.

phosphoprotein that has a role in apoptosis. Aberrantly high or deregulated Myc activity is usually implicated in various types of cancer, and is often associated with aggressive and poorly differentiated tumors (28). Cell-autonomous apoptosis following Myc overexpression has been regarded as a major tumor-suppression mechanism (29). Romero et al (30) reported that amplifications of Myc are one of the most consistent gene alterations detected in lung cancer. In the current study, overexpression of Myc was observed in the miR-1284 inhibition group, which also had the lowest apoptosis rate, suggesting that Myc may be associated with apoptosis in these cells. Additionally, the results of the present study also indicated that miR-1284 may induce apoptosis by targeting Myc in A549 lung cancer cells.

In conclusion, the current study suggests that miR-1284 affects cell proliferation, growth and apoptosis of lung cancer cells, indicating that miR-1284 may have a key role in lung tumorigenesis. Thus, miR-1284 may be a potential therapeutic agent for the treatment of lung cancer.

\section{References}

1. Peng Y, Dai Y, Hitchcock C, Yang X, Kassis ES, Liu L, Luo Z, Sun HL, Cui R, Wei $\mathrm{H}$, et al: Insulin growth factor signaling is regulated by microRNA-486, an underexpressed microRNA in lung cancer. Proc Natl Acad Sci USA 110: 15043-15058, 2013.

2. Siegel R, Naishadham D and Jemal A: Cancer statistics, 2012. CA Cancer J Clin 62: 10-29, 2012.
3. Torre LA, Bray F, Siegel RL, Ferlay J, Lortettieulent J and Jemal A: Global cancer statistics, 2012. CA Cancer J Clin 65: 87-108, 2015

4. Chen W, Zheng R, Zeng H and Zhang S: Epidemiology of lung cancer in China. Thoracic Cancer 6: 209, 2015.

5. Janssen-Heijnen ML and Coebergh JW: The changing epidemiology of lung cancer in Europe. Lung Cancer 41: 245-258, 2003.

6. Mahapatra A. Lung cancer-genomics and personalized medicine. ACS Chem Biol 5: 529-531, 2010.

7. Dumortier O and Van Obberghen E: MicroRNAs in pancreas development. Diabetes Obes Metab 14 (Suppl 3): S22-S28, 2012.

8. Croce CM: Causes and consequences of microRNA dysregulation in cancer. Nat Rev Genet 10: 704-714, 2009.

9. Nana-Sinkam SP and Croce CM: Clinical applications for microRNAs in cancer. Clin Pharmacol Ther 93: 98-104, 2013.

10. Arner P and Kulyté A: MicroRNA regulatory networks in human adipose tissue and obesity. Nat Rev Endocrinol 11: 276-288, 2015.

11. Patnaik SK, Yendamuri S, Kannisto E, Kucharczuk JC, Singhal S and Vachani A: MicroRNA expression profiles of whole blood in lung adenocarcinoma. Plos One 7: e46045, 2012.

12. Shen J and Jiang F: Applications of microRNAs in the diagnosis and prognosis of lung cancer. Expert Opin Med Diagn 6: 197-207, 2012.

13. Sun D, Li X, Ma M, Liu J, Xu Y, Ye L, Hou H, Wang C, Li X and Jiang Y: The predictive value and potential mechanisms of miRNA-328 and miRNA-378 for brain metastases in operable and advanced non-small-cell lung cancer. Jpn J Clin Oncol 45: 464-473, 2015.

14. Chen Z, Zeng H, Guo Y, Liu P, Pan H, Deng A and Hu J: miRNA-145 inhibits non-small cell lung cancer cell proliferation by targeting c-Myc. J Exp Clin Cancer Res 29: 151, 2010.

15. Liu ZL, Wang H, Liu J and Wang ZX: MicroRNA-21 (miR-21) expression promotes growth, metastasis and chemo- or radioresistance in non-small cell lung cancer cells by targeting PTEN. Mol Cell Biochem 372: 35-45, 2013.

16. Cao W, Wei W, Zhan Z, Xie Y and Xiao Q: miR-1284 modulates multidrug resistance of gastric cancer cells by targeting EIF4A1. Oncol Rep 35: 2583-2591, 2016. 
17. Livak KJ and Schmittgen TD: Analysis of relative gene expression data using real-time quantitative PCR and the 2(-Delta Delta C(T)) method. Methods 25: 402-408, 2001

18. Fernandez S, Risolino M, Mandia N, Talotta F, Soini Y, Incoronato M, Condorelli G, Banfi S and Verde P: miR-340 inhibits tumor cell proliferation and induces apoptosis by targeting multiple negative regulators of p27 in non-small cell lung cancer. Oncogene 34: 3240-3250, 2015.

19. Chu I, Hengst L and Slingerland J: The Cdk inhibitor p27 in human cancer: Prognostic potential and relevance to anticancer therapy. Nat Rev Cancer 8: 253-267, 2008.

20. Fuchs Y and Steller H: Programmed cell death in animal development and disease. Cell 147: 742-758, 2011.

21. Mckenzie S and Kyprianou N: Apoptosis evasion: The role of survival pathways in prostate cancer progression and therapeutic resistance. J Cell Biochem 97: 18-32, 2006.

22. Green DR: Apoptotic pathways: Paper wraps stone blunts scissors. Cell 102: 1-4, 2000.

23. Pop C and Salvesen GS: Human caspases: Activation, specificity, and regulation. J Biol Chem 284: 21777-217817, 2009.

24. Brentnall M, Rodriguez-Menocal L, De Guevara RL, Cepero E and Boise LH: Caspase-9, caspase-3 and caspase-7 have distinct roles during intrinsic apoptosis. BMC Cell Biol 14: 32, 2013.

25. Maurya SK, Tewari M, Sharma B and Shukla HS: Expression of procaspase 3 and activated caspase 3 and its relevance in hormone-responsive gallbladder carcinoma chemotherapy. Korean J Intern Med 28: 573-578, 2013.
26. Youle R and Strasser A: The BCL-2 protein family: Opposing activities that mediate cell death. Nat Rev Mol Cell Biol 9: 47-59, 2008.

27. Ding J, Mooers BH, Zhang Z, Kale J, Falcone D, McNichol J, Huang B, Zhang XC, Xing C, Andrews DW and Lin J: After embedding in membranes antiapoptotic Bcl-XL protein binds both Bcl-2 homology region 3 and helix 1 of proapoptotic Bax protein to inhibit apoptotic mitochondrial permeabilization. J Biol Chem 289: 11873-11896, 2014.

28. Soucek L, Whitfield JR, Sodir NM, Massó-Vallés D, Serrano E, Karnezis AN, Swigart LB and Evan GI: Inhibition of Myc family proteins eradicates KRas-driven lung cancer in mice. Genes Dev 27: 504-513, 2013.

29. Meyer N, Kim SS and Penn LZ: The Oscar-worthy role of Myc in apoptosis. Semin Cancer Biol 16: 275-287, 2006.

30. Romero OA, Torres-Diz M, Pros E, Savola S, Gomez A, Moran S, Saez C, Iwakawa R, Villanueva A, Montuenga LM, et al: MAX inactivation in small cell lung cancer disrupts MYC-SWI/SNF programs and is synthetic lethal with BRG1. Cancer Discov 4: 292-303, 2014. 